\title{
Cubosomes as Oral Drug Delivery Systems: A Promising Approach for Enhancing the Release of Clopidogrel Bisulphate in the Intestine
}

\author{
Hanan M. El-Laithy, ${ }^{a, b}$ Alia Badawi, ${ }^{a}$ Nevine Shawky Abdelmalak, ${ }^{a}$ and Nihal El-Sayyad ${ }^{*, b}$ \\ ${ }^{a}$ Department of Pharmaceutics and Industrial Pharmacy, Faculty of Pharmacy, Cairo University; Cairo 11562, \\ Egypt: and ${ }^{b}$ Department of Pharmaceutics and Industrial Pharmacy, Faculty of Pharmacy, October University for \\ Modern Sciences and Arts (MSA); 6th of October 12582, Egypt. \\ Received August 9, 2018; accepted September 6, 2018; advance publication released online September 19, 2018
}

\begin{abstract}
Clopidogrel bisulphate (CB) is a first line antiplatelet drug for treatment of myocardial infarction and stroke. Yet, its efficacy is limited by its poor solubility in intestinal $\mathrm{pH}$, its main site of absorption. The main aim of this study is to enhance the intestinal release of $\mathrm{CB}$ by loading in cubosome nanoparticles. Glyceryl monooleate (GMO) based CB loaded cubosomes were prepared using a $3^{3}$ factorial design to study the effect of polyvinyl alcohol (PVA), poloxamer 407 (PL407) concentrations and ratio of CB to the disperse phase on the average particle size, entrapment efficiency $(\% \mathrm{EE})$, in vitro release at $15 \mathrm{~min}\left(\% \mathrm{Q}_{15}\right)$, and their morphology using transmission electron microscopy (TEM). The release of the optimized formula was compared in buffer transition media ( $\mathrm{pH} 1.2$ for $2 \mathrm{~h}$ then $\mathrm{pH} 6.8$ for $6 \mathrm{~h}$ ) to free drug to study the effect of the changing $\mathrm{pH}$ in the gastrointestinal tract (GIT) on CB release. The antihaemostatic properties of the optimized formula were compared to the commercial product Plavix ${ }^{\circledR}$ using bleeding time (BT) model in rabbits. The prepared cubosomes were in the nano range $(115 \pm 6.47$ to $248 \pm 4.63 \mathrm{~nm})$ with high \%EE $(91.22 \pm 4.09 \%$ to $98.98 \pm 3.21 \%)$. The optimized formula showed significantly higher $(p<0.05)$ CB release in intestinal pH and preserved the high\% released $(95.66 \pm 1.87 \%)$ in buffer transition release study compared to free drug $(66.82 \pm 4.12 \%)$ as well as significantly $(p<0.05)$ higher antihaemostatic properties with longer BT (628.47 $\pm 6.12 \mathrm{~s})$ compared to Plavix ${ }^{\circledR}(412.43 \pm 7.97 \mathrm{~s})$. Thus, cubosomes proved to be a successful platform to enhance the intestinal release of $\mathrm{CB}$ and improve its absorption.
\end{abstract}

Key words clopidogrel bisulphate; cubosome; factorial design; nanoparticle; buffer transition release study; bleeding time

Clopidogrel bisulphate (CB) is a thienopyridene class antiplatelet oral drug for the treatment of patients with a high risk of myocardial infarction and stroke. ${ }^{1)} \mathrm{CB}$ is a prodrug that is absorbed in the intestine and activated in the liver. ${ }^{2)}$ It is absorbed rapidly following oral administration, with mean halflife of active metabolite in a range of $7-10 \mathrm{~h} .{ }^{3)}$ Clopidogrel is an inactive prodrug which undergoes CYP3A4-dependent metabolism before exhibiting its antiaggregatory effect. ${ }^{4)} \mathrm{CB}$ acts by selectively and irreversibly inhibiting ADP-induced platelet activation and aggregation via blocking the binding of ADP to the purinergic P2Y12 receptor located on the platelet surface. ${ }^{5}$

$\mathrm{CB}$ is categorized as biopharmaceutics classification system (BCS) class II drug, this is primarily due to its poor solubility in intestinal $\mathrm{pH}^{6}$ the main site of its absorption ${ }^{7)}$ resulting in very low $(<50 \%)$ oral bioavailability. ${ }^{8)}$ Following oral administration, the high solubility of $\mathrm{CB}$ in gastric $\mathrm{pH}$, causes its protonation and ionization and thus hampers its absorption from the upper gastrointestinal tract (GIT). ${ }^{6)}$ Once in the intestine, its poor solubility in intestinal $\mathrm{pH}$ causes it to precipitate and consequently diminishes its absorption and bioavailability $^{7}$ Accordingly, if the intestinal solubility and the release properties of $\mathrm{CB}$ is improved, its absorption and consequently its in vivo bioavailability will be enhanced. ${ }^{9}$ )

Lipid based nanoparticles are considered to be one of the most successful strategies to enhance the dissolution and permeability of drugs. The drug is dissolved, entrapped, encapsulated or attached to a nanoparticle matrix thereby remarkably influencing release profiles of drugs. ${ }^{10)}$ Amongst these drug delivery systems are cubosome nanoparticles. Cubosomes are unique and intriguing self-assembled nanoparticles with enormous potential in diverse areas as medicine, materials science, and consumer products. Cubosomes are formed by amphiphilic or surfactant like molecules which self-assemble into the complex three-dimensional cubic phase structure through the self-organization into bilayers around bicontinuous non-intersecting water channels ${ }^{11)}$ and these structures can be harnessed to encapsulate hydrophilic, hydrophobic and amphiphilic molecules. ${ }^{12}$ They also possess excellent loading properties, ${ }^{13)}$ facilitate absorption ${ }^{14,15)}$ and offer protection for drugs against degradation ${ }^{16,17)}$ thus making them an excellent choice for formulation of poorly soluble drugs orally. ${ }^{18,19)}$ Cubosomes can maintain the drug in a solubilized state in the gastrointestinal tract by entrapping drugs into the mixed micelles produced by the digestion of cubosomes, thereby enhance drug release and absorption leading to improved oral bioavailability. ${ }^{13,20)}$

Therefore, based on the above considerations, the main aim of this work is to enhance the solubility and the in vitro release of $\mathrm{CB}$ through the formulation of oral cubosome nanoparticles. A $3^{3}$ full factorial design was applied to study the effects of different formulation factors on particle size, entrapment efficiency $(\% \mathrm{EE})$ and percentage of $\mathrm{CB}$ release at 15 min in intestinal $\mathrm{pH}\left(\% \mathrm{Q}_{15}\right)$ of the resultant formulas. The pharmacological effect of the optimized formula was assessed by comparing its antihaemostatic properties through bleeding time (BT) measurements in rabbits to the market product Plavix ${ }^{\circledR}$ as an indicator of improved CB levels in blood stream and enhanced drug efficacy. 


\section{Experimental}

Materials Clopidogrel bisulfate (CB) was obtained as a gift from Eva pharma, Egypt. Glyceryl monooleate (GMO), poloxamer 407 (PL407), polyvinyl alcohol (PVA) were purchased from Sigma-Aldrich (St. Louis, MO, U.S.A.). Spectra/ Pore dialysis membrane (12000-14000 molecular weight cutoff) was purchased from Spectrum Laboratories Inc. (U.S.A.). The commercial tablet Plavix ${ }^{\circledR}$ (Sanofi, France) was purchased to be used in comparative studies. All other chemicals used were of analytical grade and were obtained from standard commercial suppliers.

Determination of CB Solubility in Different $\mathrm{pH}$ Media Excess amount of $\mathrm{CB}$ was mixed with $100 \mathrm{~mL}$ of each of $\mathrm{HCl}$ buffer ( $\mathrm{pH}$ 1.2), $\mathrm{HCl}$ buffer $(\mathrm{pH} 2.0)$, phosphate buffer $(\mathrm{pH}$ 5.5), phosphate buffer ( $\mathrm{pH} 6.8)$, phosphate buffer ( $\mathrm{pH} 7.4)$ and phosphate buffer ( $\mathrm{pH}$ 6.8) with $1 \%$ Sodium Lauryl Sulphate (SLS). The solutions were sonicated for $48 \mathrm{~h}$ at $37 \pm 2{ }^{\circ} \mathrm{C}$. The resulting solutions were left for $24 \mathrm{~h}$ to allow excess amounts to precipitate. The non-dissolved drug was filtered and the supernatant was analyzed by HPLC method ${ }^{21)}$ using an Agilent 1100 HPLC system (Agilent, U.S.A.) with a UV detector (Agilent VWD G1314A, U.S.A.). Chromatographic separation was carried out on Nova-Pak C18 column $(5 \mu \mathrm{m}, 4.6 \times 150 \mathrm{~mm}$, Waters, U.S.A.) maintained at $35^{\circ} \mathrm{C}$. The mobile phase consisted of $60 \%$ acetonitrile and $40 \%$ of $0.05 \mathrm{M}$ sodium hydrogen phosphate adjusted to $\mathrm{pH} 4$ using phosphoric acid. The mobile phase was filtered through $0.2 \mu \mathrm{m}$ membrane filter and delivered at a flow rate of $1 \mathrm{~mL} / \mathrm{min}$. Samples of $10 \mu \mathrm{L}$ were injected and effluents were monitored at $220 \mathrm{~nm}$.

Preparation of CB Loaded Cubosome Dispersions CB loaded cubosome dispersions were prepared according to the method described by Morsi et al. ${ }^{22)}$ First GMO and PL407 were melted on a hot plate at temperature $70 \pm 2{ }^{\circ} \mathrm{C}$. Clopidogrel was dispersed in the molten mixture. PVA was dissolved in $2 \mathrm{~mL}$ distilled water at $80^{\circ} \mathrm{C}$ then was added dropwise to the molten mixture and thoroughly mixed at $1500 \mathrm{rpm}$ with magnetic stirrer (Thermo Scientific, U.S.A.) till cubic gel was formed. To form the cubosome dispersions, distilled water was added dropwise to the cubic gel at $70 \pm 2^{\circ} \mathrm{C}$ under mechanical stirring so that the disperse phase (molten mixture) would constitute $10 \%$ from the final dispersion. Dispersions were maintained under stirring and were cooled to room temperature and then stored in glass bottles at room temperature for further investigations.

Statistical Design of the Study A $3^{3}$ full factorial experimental design was applied to investigate the influence of the formulation variables such as PL407 concentration $(2.5,5$, $10 \%)$, PVA concentration $(0,2.5,5 \%)$ as well as the drug to the disperse phase ratio $(1: 10,2: 10,3: 10)$ on the characteristics of cubosome dispersions (dependent variables) such as particle size, entrapment efficiency $(\% \mathrm{EE})$ and in vitro drug dissolved at $15 \mathrm{~min}\left(\% \mathrm{Q}_{15}\right)$ using Minitab $17^{\circledR}$ software (version 17, Minitab Inc., U.S.A.). The composition of different formulations (CL1-CL27) is listed in Table 1.

Data collected for each response of the dependent variables from the 27 runs was analyzed using first order multiple linear regression model by fitting into the following formula. ${ }^{23)}$

$$
Y=\beta_{\mathrm{o}}+\sum \beta_{\mathrm{i}} X_{\mathrm{i}}
$$

Where $Y$ is the measured response associated with each factor level combinations, $\beta_{\mathrm{o}}$ is the model intercept, $\beta_{\mathrm{i}}$ is the linear coefficient and $X_{\mathrm{i}}$ is the level of the independent variable.

The first-order polynomial in Eq. 1 was fitted to raw responses. ANOVA was used to evaluate the statistical significance of the model. Level of significance was set at $(\alpha=0.05)$. The influence of response variable transformation was investigated by fitting Eq. 1 to log, reciprocal, square root and square of transformed response variables and comparing two statistical parameters: the adjusted multiple correlation coefficient (adjusted $R^{2}$ ) and the $p$ values for the overall $F$-ratio test of fit adequacy. ${ }^{24)}$ Main effects plots and interaction plots were obtained and visually inspected.

Additionally, desirability values were calculated for the optimization and selection of the optimized formula with the highest desirability value. According to Derringer and Suich, ${ }^{25}$ desirability index (D) is a score between 0 and 1 reflecting the level of satisfaction with a given combination of independent variables. D of 0 corresponds to a completely undesirable formulation, and $\mathrm{D}$ of 1 corresponds to a completely desirable one.

If maximization of a response $Y$ is wanted, desirability is calculated for each $Y$ value according to the following equation:

$$
\mathrm{Dy}=\left\{\begin{array}{ccc}
\frac{Y-Y_{\min }}{Y_{\max }-Y_{\min }} & \text { for } & Y_{\min } \leq Y \leq Y_{\max } \\
0 & & \text { otherwise }
\end{array}\right.
$$

If minimization of $Y$ is wanted:

Table 1. Composition of Different Prepared CB Loaded Cubosome Dis-

\begin{tabular}{|c|c|c|c|c|}
\hline \multirow{2}{*}{ Formula } & \multicolumn{3}{|c|}{ Disperse phase } & \multirow{2}{*}{$\begin{array}{l}\text { Ratio of drug to } \\
\text { disperse phase }\end{array}$} \\
\hline & GMO (\%) & PL407 (\%) & PVA (\%) & \\
\hline CL1 & 97.5 & 2.5 & 0 & $1: 10$ \\
\hline CL2 & 95 & 2.5 & 2.5 & $1: 10$ \\
\hline CL3 & 92.5 & 2.5 & 5 & $1: 10$ \\
\hline CL4 & 95 & 5 & 0 & $1: 10$ \\
\hline CL5 & 92.5 & 5 & 2.5 & $1: 10$ \\
\hline CL6 & 90 & 5 & 5 & $1: 10$ \\
\hline CL7 & 90 & 10 & 0 & $1: 10$ \\
\hline CL8 & 87.5 & 10 & 2.5 & $1: 10$ \\
\hline CL9 & 85 & 10 & 5 & $1: 10$ \\
\hline CL10 & 97.5 & 2.5 & 0 & $2: 10$ \\
\hline CL11 & 95 & 2.5 & 2.5 & $2: 10$ \\
\hline CL12 & 92.5 & 2.5 & 5 & $2: 10$ \\
\hline CL13 & 95 & 5 & 0 & $2: 10$ \\
\hline CL14 & 92.5 & 5 & 2.5 & $2: 10$ \\
\hline CL15 & 90 & 5 & 5 & $2: 10$ \\
\hline CL16 & 90 & 10 & 0 & $2: 10$ \\
\hline CL17 & 87.5 & 10 & 2.5 & $2: 10$ \\
\hline CL18 & 85 & 10 & 5 & $2: 10$ \\
\hline CL19 & 97.5 & 2.5 & 0 & $3: 10$ \\
\hline CL20 & 95 & 2.5 & 2.5 & $3: 10$ \\
\hline CL21 & 92.5 & 2.5 & 5 & $3: 10$ \\
\hline CL22 & 95 & 5 & 0 & $3: 10$ \\
\hline CL23 & 92.5 & 5 & 2.5 & $3: 10$ \\
\hline CL24 & 90 & 5 & 5 & $3: 10$ \\
\hline CL25 & 90 & 10 & 0 & $3: 10$ \\
\hline CL26 & 87.5 & 10 & 2.5 & $3: 10$ \\
\hline CL27 & 85 & 10 & 5 & $3: 10$ \\
\hline
\end{tabular}
persions 


$$
\mathrm{Dy}=\left\{\begin{array}{ccl}
\frac{Y_{\max }-Y}{Y_{\max }-Y_{\min }} & \text { for } & Y_{\min } \leq Y \leq Y_{\max } \\
0 & & \text { otherwise }
\end{array}\right.
$$

If a target response, $Y_{\operatorname{targ}}$, is wanted:

$$
\mathrm{Dy}=\left\{\begin{array}{ccc}
1-\frac{Y-Y_{\mathrm{targ}}}{Y_{\max }-Y_{\min }} & \text { for } & Y_{\mathrm{targ}} \leq Y \leq Y_{\max } \\
1-\frac{Y_{\mathrm{targ}}-Y}{Y_{\max }-Y_{\min }} & \text { for } & Y_{\min } \leq Y \leq Y_{\mathrm{targ}} \\
0 & & \text { otherwise }
\end{array}\right.
$$

where $Y_{\min }$ and $Y_{\max }$ define ranges of acceptable response values. When multiple responses are present, individual desirability index must be calculated for each response, then a joint desirability $\left(\mathrm{D}_{\text {joint }}\right)$ is defined as the weighted geometric mean of individual desirability indices normalized by the number of responses $n$ :

$$
\mathrm{D}_{\text {joint }}=\left[\prod_{i=1}^{n} \mathrm{D}_{Y_{\mathrm{i}}}^{W_{\mathrm{i}}}\right]^{1 / n}
$$

where $W_{\mathrm{i}}$ are relative importance weights of different responses (the weights must add up to 1).

In this study, the criteria set for optimal formulation selection was achieving the least particle size associated with the maximum $\% \mathrm{EE}$ and the highest $\% \mathrm{Q} 15$. The three criteria were considered equally important; therefore, the weights were set to $1 / 3$.

Transmission Electron Microscopy (TEM) One drop of the cubosome dispersion was deposited on carbon-coated copper grid (200 mesh) and negatively stained using phosphotungstic acid $(1 \% \mathrm{w} / \mathrm{v})$ with the excess stain removed using a filter paper. The grid was completely dried at ambient temperature and the measurements were performed with a TEM microscope (JEM-1230, Jeol, Tokyo, Japan).

Particle Size The particle size of the cubosomal dispersions were determined by using photon correlation spectroscopy. Samples were diluted (100-fold) with de-ionized water and placed in a scattering chamber where the light scattering was monitored at $90^{\circ}$ scattering angle using a Zetasizer Nano ZS (Malvern Instruments Ltd., Worcestershire, U.K.). All measurements were performed at $25 \pm 0.5^{\circ} \mathrm{C}$ in triplicate.

\%EE CB loaded cubosomes were separated from unentrapped drug by centrifuging $2 \mathrm{~mL}$ of $\mathrm{CB}$ cubosomal dispersion in eppendorf tube at $12000 \mathrm{rpm}$ for $30 \mathrm{~min}$ at $4^{\circ} \mathrm{C}$ (centrifuge model 5417R, eppendorf, Hamburg, Germany). The supernatant was diluted with the mobile phase and the free unentrapped $\mathrm{CB}$ concentration in the resulting solution was assayed by HPLC method mentioned earlier. The entrapment efficiency was calculated using the following equation ${ }^{26)}$

$$
(\% \mathrm{EE})=\frac{\begin{array}{c}
\text { Total CB in dispersion } \\
- \text { Free unentrapoed } \mathrm{CB} \text { in dispersion }
\end{array}}{\text { Total CB in dispersion }} \times 100
$$

In Vitro Drug Release Studies The in vitro release of $\mathrm{CB}$ from the cubosome dispersion was determined using United States Pharmacopeia (USP) dissolution tester, apparatus II (Erweka, Germany). Cubosome dispersion containing the equivalent of $97.8 \mathrm{mg} \mathrm{CB}$ was placed in a Spectra/Por ${ }^{\circledR} \mathrm{di}$ - alysis membrane of 12000-14000 molecular weight cutoff and sealed. The membrane was washed with distilled water several times to remove preservative and soaked in release medium overnight before use. The dispersion filled membrane was introduced into the dissolution apparatus cups using $1000 \mathrm{~mL}$ phosphate buffer $\mathrm{pH} 6.8$ containing $1 \%$ SLS to maintain sink conditions due to poor solubility of $\mathrm{CB}$ in phosphate buffer. ${ }^{27)}$ The release study was carried out at $37 \pm 0.5^{\circ} \mathrm{C}$, and the stirring shafts were rotated at a speed of $100 \mathrm{rpm}$. Three milliliter samples were withdrawn periodically at predetermined time intervals of 5, 10, 15, 30, 45 and $60 \mathrm{~min}$ and replaced instantly by equal amount of fresh release medium to maintain a constant volume. Samples were filtered using $0.22 \mu \mathrm{m}$ filter and analyzed by HPLC method previously described. All release experiments were done in triplicates.

The release of the optimized $\mathrm{CB}$ cubosome dispersion and free $\mathrm{CB}$ powder were compared using a buffer transition release media to assess the effect of changing $\mathrm{pH}$ conditions on $\mathrm{CB}$ release through its movement along human GIT. The release study was done in a USP type II apparatus rotated at $100 \mathrm{rpm}$ at a temperature of $37 \pm 0.5^{\circ} \mathrm{C}$. The acid stage comprised the use of $750 \mathrm{~mL} 0.1 \mathrm{~N} \mathrm{HCl}$ buffer as a release medium for $2 \mathrm{~h}$, after which the $\mathrm{pH}$ was adjusted to $\mathrm{pH} 6.8$ with $250 \mathrm{~mL} 0.2 \mathrm{M}$ sodium triphosphate buffer ${ }^{21,28,29)}$ and the experiment was continued for $6 \mathrm{~h}$. One percent SLS was added to achieve sink conditions in $\mathrm{pH}$ 6.8. Samples were withdrawn at $0.25,0.5,0.5,1,1.5,2,2.5,3,4,6$ and $8 \mathrm{~h}$, filtered and analyzed using the same method described earlier The release profiles were compared using similarity factor (f2) defined by the following equation ${ }^{30}$ )

$$
\mathrm{f} 2=50 \log \left\{\left[1+\frac{1}{n} \sum_{t=1}^{n} R_{\mathrm{t}}-T_{\mathrm{t}}\right]^{-0.5}\right\} \times 100
$$

Where, $n=$ the number of sampling time points, $R_{\mathrm{t}}=$ mean $\%$ released of the reference at a given time point $t, T_{\mathrm{t}}=$ mean $\%$ released of the test at the same time point $t$. The similarity factor fits the result between 0 and 100. It is 100 when the two release profiles are identical. ${ }^{31)}$ In order to consider similar release profiles, f2 values should be higher than 50, whereas smaller values imply an increase in the dissimilarity between release profiles over all time points.

In Vivo BT The blood clotting properties are considered as an indicator for the extent of absorption of $\mathrm{CB}$ from the optimized cubosomal dispersion compared to the commercial marketed Plavix ${ }^{\circledR}$ tablet using a parallel randomized design. The protocol of the study was reviewed and approved by the Research Ethics Committee (protocol serial number PI 1387) at Faculty of Pharmacy, Cairo University (Cairo, Egypt) and the rabbit cuticle BT model described by Wong et al. ${ }^{32)}$ was used. Eighteen male New Zealand white rabbits weighing $2.5 \pm 0.11 \mathrm{~kg}$ each were randomly distributed between three groups each of six rabbits. The rabbits were housed individually in stainless steel cages and fed a commercial diet. In a dose of $20 \mathrm{mg} / \mathrm{kg}$, group 1 received the optimized formula CL1 while group 2 was administered the marketed product (Plavix $^{\circledR}$, Sanofi Aventis, Cairo, Egypt) by oral gavage with $1 \mathrm{~mL}$ water. ${ }^{33)}$ Group 3 served as a control and did not receive any treatments. Two hours after the administration, the animals were anesthetized with ketamine $(50 \mathrm{mg} / \mathrm{kg}+50 \mathrm{mg} / \mathrm{kg} / \mathrm{h}$ Intra-muscular (IM)) and xylazine $(10 \mathrm{mg} / \mathrm{kg}+10 \mathrm{mg} / \mathrm{kg} / \mathrm{h}$ 
IM). ${ }^{32)}$ The anesthetized rabbits hind paws were shaved and a standard cut was made at the apex of the cuticle with a razor blade. Blood was allowed to flow freely by keeping the bleeding site in contact with $37^{\circ} \mathrm{C}$ lactated Ringer's solution. BT, which is defined as the time after transection when bleeding ceased was measured and the average was calculated.

Statistical Analysis The data obtained from different tests were analyzed for statistical significance by one-way ANOVA using Minitab ${ }^{\circledR}$ software (version 17, Minitab Inc., U.S.A.) followed by post hoc multiple comparisons using the least square difference (LSD). Differences were considered to be significant at $p \leq 0.05$.

\section{Results and Discussion}

Determination of CB Solubility in Different pH Media Solubility studies were done to confirm the poor solubility of $\mathrm{CB}$ in intestinal $\mathrm{pH}$ conditions and to select the appropriate release medium in this study. As shown in Table 2, CB is freely soluble in lower $\mathrm{pH}$ values while practically insoluble in water and higher $\mathrm{pH}$ which comes in agreement with previous studies. ${ }^{27)} \mathrm{A}$ direct relationship is evident between $\mathrm{pH}$ and solubility of $\mathrm{CB}$, with solubility decreasing as the $\mathrm{pH}$ increases which confirms the poor solubility of $\mathrm{CB}$ in intestinal $\mathrm{pH}$ (6.8) and accounts for its poor bioavailability. Addition of $1 \%$ SLS to phosphate buffer ( $\mathrm{pH}$ 6.8) resulted in marked increase in $\mathrm{CB}$ solubility in this buffer, thereby would be added to the release medium in this study to achieve sink conditions.

Preparation of CB Loaded Cubosome Dispersions According to Table 1, twenty seven clopidogrel loaded cubosomal dispersions were successfully prepared (CL1 to CL27). All formulas gave transparent gel prior to the addition of water. After the addition of water they gave milky dispersions. GMO was used in the preparation of cubosomes in this study due to its ability to spontaneously form cubic phases upon mixing it with water, ${ }^{34)}$ in addition to being a safe, non-toxic, biocompatible and biodegradable ester. ${ }^{16)}$ PL407 and PVA were used as a surfactant and stabilizer respectively. ${ }^{22)}$

TEM The morphology of the cubosome dispersion investigated using TEM in Fig. 1 clearly confirms the formation of cubosome nanoparticles. Micrographs also demonstrate that the prepared cubosomes are in the nano range, non-aggregated and well separated from each other.

Particle Size The particle size calculated from the TEM images is comparable and in good agreement with that measured by the Zetasizer (Table 3) ensuring that all the prepared formulae successfully yielded cubosome particles in the nano range between $115 \pm 6.47$ to $248 \pm 4.63 \mathrm{~nm}$.

By examining the main effects of factors tested on particle size, it is clear from Fig. 2B that the particle size is mostly affected by the ratio of the drug to the disperse phase with

Table 2. Solubility of Clopidogrel Bisulphate in Different $\mathrm{pH}$ Media

\begin{tabular}{lc}
\hline \hline Solvent & Solubility (mg/L) \\
\hline $\mathrm{HCl}$ Buffer (pH 1.2) & $594.23 \pm 4.23$ \\
$\mathrm{HCl}$ buffer (pH 2.0) & $543.72 \pm 14.46$ \\
Phosphate buffer (pH 5.5) & $21.75 \pm 7.56$ \\
Phosphate buffer (pH 6.8) & $19.5 \pm 4.53$ \\
Phosphate buffer (7.4) & $15.4 \pm 2.42$ \\
Phosphate buffer (pH 6.8) with 1\% SLS & $408.23 \pm 24.23$ \\
\hline
\end{tabular}

significant increase $(p<0.05)$ in particle size when the ratio increases (Fig. 2A). This finding comes in line with previous reports. ${ }^{13,35)}$ On the contrary, it is clear from Fig. 2A, that increasing PL407 concentration significantly $(p<0.05)$ decreased the particle size. This can be attributed to the ability of PL407 as a surfactant to decrease the surface tension and consequently decrease the surface energy of the cubosome nanoparticles, thereby preventing particle aggregation ${ }^{36)}$ and decreasing particle size. ${ }^{37)}$ These findings also endorse PL407 in cubosome formulations as the main responsible ingredient for its stability. ${ }^{38)}$ Interestingly it was observed that increasing PVA concentration had insignificant effect on particle size $(p>0.05)$. Significant interactions were absent between factors tested as shown in Fig. 2C confirmed by two way ANOVA $(p>0.05)$

\%EE All the prepared cubosome nanoparticles achieved successfully entrapped $\mathrm{CB}$ with high \%EE ranging from $91.22 \pm 4.09$ to $98.98 \pm 3.21 \%$ (Table 3 ). This success can be attributed to the lipophilic nature of $\mathrm{CB}^{39)}$ which causes it to possess high affinity to the hydrophobic region of the cubic phase. $^{13)}$

As evident from the main effects plot (Fig. 3A) and pareto plot (Fig. 3B), the $\% \mathrm{EE}$ was only affected by the drug to the disperse phase ratio where the \%EE significantly $(p<0.05)$ decreased as the ratio of the drug to the disperse phase was increased. This pattern could be attributed to the saturation of the bulk cubic phase with the drug due to its lipophilic nature, thereby causing a disturbance of the bulk cubic phase making the drug escape to the aqueous medium. ${ }^{13)}$ On the contrary, both PL407 and PVA concentrations had insignificant $(p>0.05)$ effects on $\%$ EE. No significant interactions were detected between PL407 concentration and PVA concentration or PVA concentration and ratio of drug to disperse phase $(p>0.05)$ as shown in Fig. $3 \mathrm{C}$ which was confirmed by two way ANOVA. However, a significant interaction was found between PL407 concentration and the ratio of drug to disperse phase confirmed by two way ANOVA $(p<0.05)$. This interaction can be explained by the effect of PL407 as a surfactant on the partitioning and the solubilization of $\mathrm{CB}$ in the hydrophobic region of the cubosome thus affecting its entrapment inside cubosome nanoparticles. ${ }^{40)}$

In Vitro Drug Release Studies As depicted from Figs. $4 \mathrm{~A}-\mathrm{C}$, all the prepared CB cubosome dispersions exhibited a biphasic release behavior in phosphate buffer containing $1 \% \mathrm{SLS}$ with a rapid burst release after only $15 \mathrm{~min}\left(\% \mathrm{Q}_{15} c a\right.$. between $58.71 \pm 7.23$ to $93.21 \pm 5.22 \%$ ) followed by a slower rate of release. This profile agrees with the previous reports

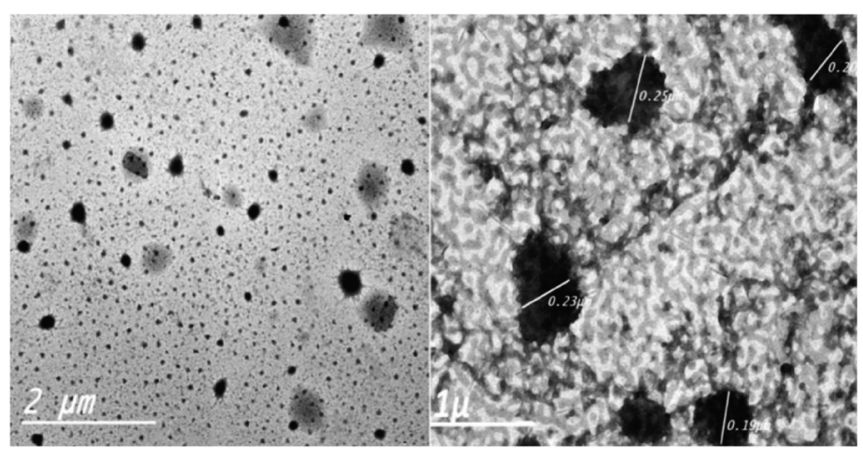

Fig. 1. TEM Micrograph of CB Cubosome Dispersion 
Table 3. The Measured Responses of Different Prepared CB Loaded Cubosome Dispersions and the Resultant Desirability Index

\begin{tabular}{|c|c|c|c|c|}
\hline Formulae & Particle size \pm S.D. (nm, $n=3$ ) & $\% \mathrm{EE} \pm$ S.D. $(n=3)$ & $\% \mathrm{Q}_{15} \pm$ S.D. $(n=6)$ & Desirability index \\
\hline CL1 & $153 \pm 4.35$ & $98.98 \pm 3.21$ & $93.21 \pm 5.22$ & 0.867668 \\
\hline CL2 & $139 \pm 7.73$ & $96.21 \pm 5.89$ & $71.15 \pm 6.47$ & 0.700690 \\
\hline CL3 & $165 \pm 5.84$ & $97.21 \pm 9.46$ & $70.49 \pm 5.77$ & 0.759330 \\
\hline CL4 & $143 \pm 8.27$ & $95.18 \pm 2.47$ & $77.64 \pm 4.12$ & 0.620976 \\
\hline CL5 & $138 \pm 8.46$ & $94.76 \pm 3.46$ & $71.42 \pm 5.77$ & 0.725396 \\
\hline CL6 & $148 \pm 4.26$ & $96.43 \pm 7.41$ & $74.13 \pm 2.31$ & 0.616462 \\
\hline CL7 & $124 \pm 9.25$ & $97.21 \pm 2.76$ & $63.08 \pm 2.12$ & 0.534747 \\
\hline CL8 & $115 \pm 6.47$ & $95.65 \pm 5.47$ & $78.41 \pm 5.47$ & 0.593242 \\
\hline CL9 & $138 \pm 9.34$ & $96.21 \pm 8.47$ & $61.39 \pm 6.77$ & 0.504888 \\
\hline CL10 & $169 \pm 5.46$ & $97.29 \pm 9.42$ & $73.95 \pm 2.84$ & 0.718047 \\
\hline CL11 & $149 \pm 9.47$ & $93.34 \pm 4.95$ & $82.98 \pm 7.51$ & 0.605815 \\
\hline CL12 & $185 \pm 4.47$ & $95.42 \pm 9.46$ & $72.17 \pm 3.43$ & 0.590942 \\
\hline CL13 & $149 \pm 5.85$ & $97.32 \pm 7.83$ & $79.71 \pm 6.78$ & 0.638576 \\
\hline CL14 & $148 \pm 6.46$ & $97.21 \pm 1.47$ & $89.27 \pm 3.58$ & 0.840669 \\
\hline CL15 & $179 \pm 4.75$ & $96.39 \pm 5.76$ & $78.32 \pm 8.39$ & 0.621149 \\
\hline CL16 & $136 \pm 4.57$ & $96.52 \pm 2.34$ & $64.34 \pm 1.92$ & 0.468708 \\
\hline CL17 & $169 \pm 4.46$ & $97.93 \pm 4.58$ & $68.56 \pm 2.85$ & 0.556770 \\
\hline CL18 & $149 \pm 5.35$ & $97.12 \pm 6.46$ & $62.56 \pm 3.09$ & 0.435011 \\
\hline CL19 & $165 \pm 6.47$ & $97.47 \pm 7.49$ & $85.88 \pm 1.89$ & 0.812696 \\
\hline CL20 & $195 \pm 5.46$ & $95.19 \pm 3.68$ & $68.64 \pm 5.24$ & 0.370924 \\
\hline CL21 & $186 \pm 7.46$ & $95.07 \pm 2.31$ & $67.86 \pm 9.53$ & 0.199958 \\
\hline CL22 & $243 \pm 4.55$ & $91.56 \pm 5.67$ & $75.05 \pm 2.08$ & 0.223551 \\
\hline CL23 & $238 \pm 6.47$ & $94.73 \pm 3.33$ & $88.91 \pm 3.81$ & 0.312010 \\
\hline CL24 & $248 \pm 4.63$ & $92.21 \pm 4.69$ & $70.48 \pm 5.86$ & 0.193021 \\
\hline CL25 & $194 \pm 2.46$ & $93.19 \pm 2.92$ & $60.41 \pm 3.04$ & 0.155169 \\
\hline CL26 & $178 \pm 3.85$ & $91.22 \pm 4.09$ & $64.43 \pm 6.67$ & 0.183717 \\
\hline CL27 & $188 \pm 4.56$ & $93.21 \pm 5.61$ & $58.71 \pm 7.23$ & 0.121166 \\
\hline
\end{tabular}

A

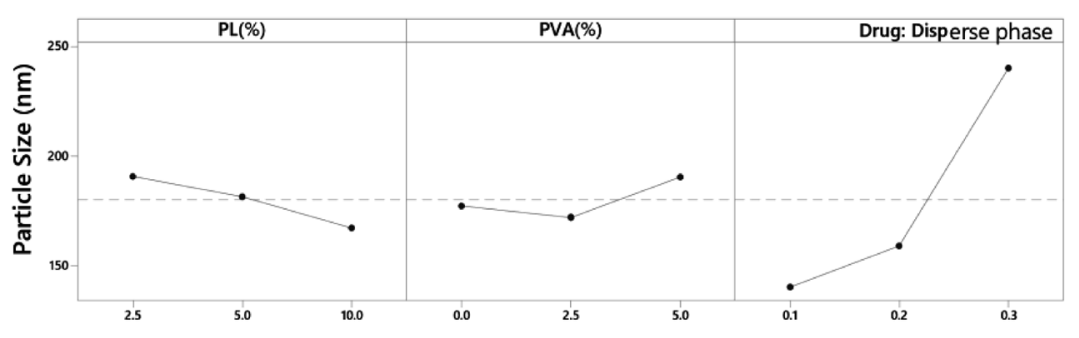

Interaction Plot
B

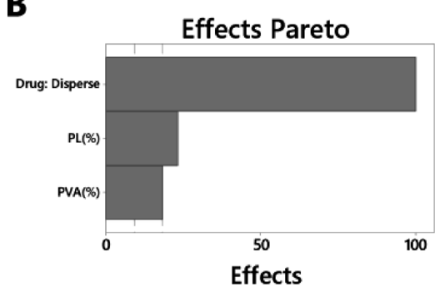

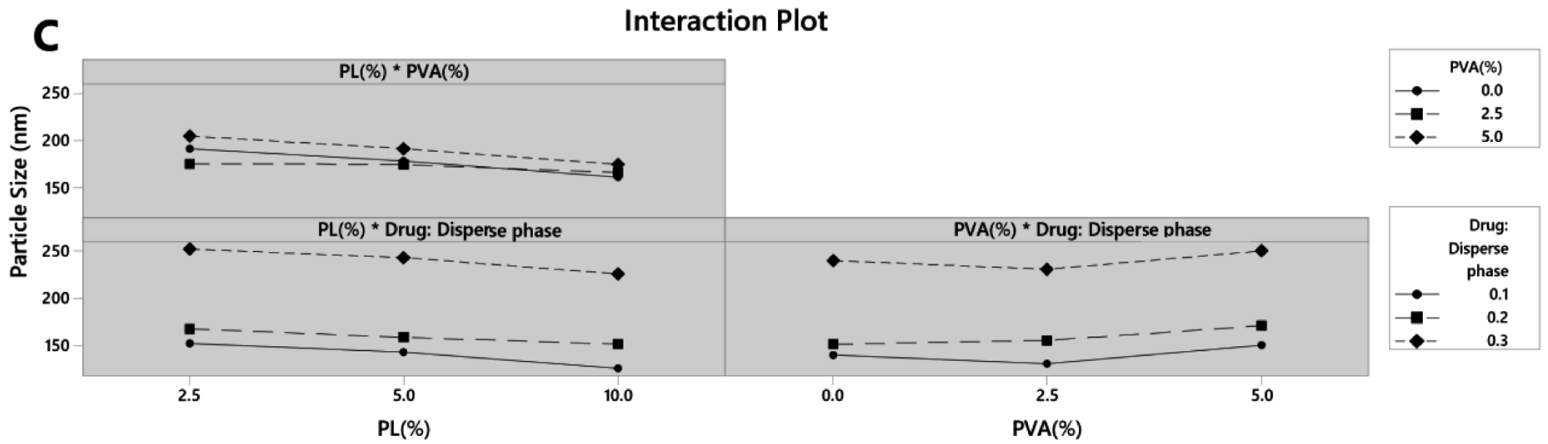

Fig. 2. Main Effect Plot, Interaction Plot and Pareto Plot of PL407 Concentration, PVA Concentration and Drug to Disperse Phase Ratio on Particle Size for CB Loaded Cubosome Dispersions

by Boyd and Nasr who studied the release of lipophilic drugs from cubosome particles. ${ }^{12,41)}$ The burst release pattern could be explained by the ability of the cubosome nanoparticles to keep the insoluble $\mathrm{CB}$ in a solubilized nano size state with the formation of concomitant large surface area for the diffusion of the drug from the nanoparticles upon exposure to the release medium in appropriate sink conditions. ${ }^{20,42,43)}$ While the tortuosity and the narrow pore size of the inner aqueous 
A

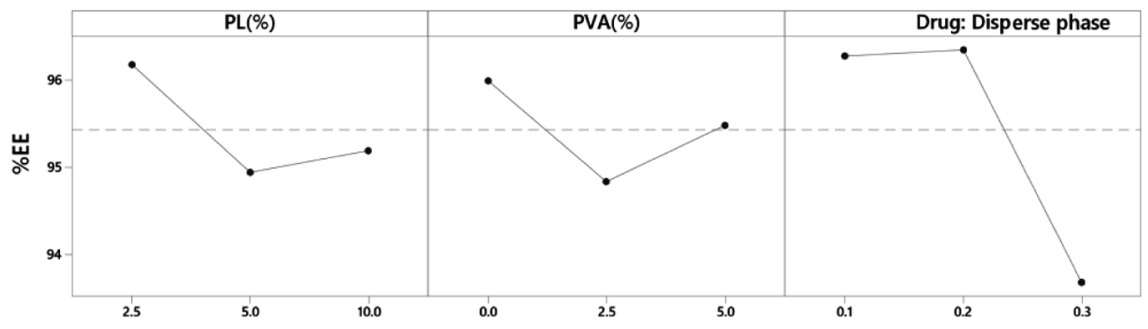

B

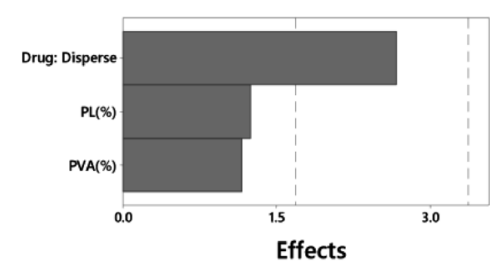

Interaction Plot

C

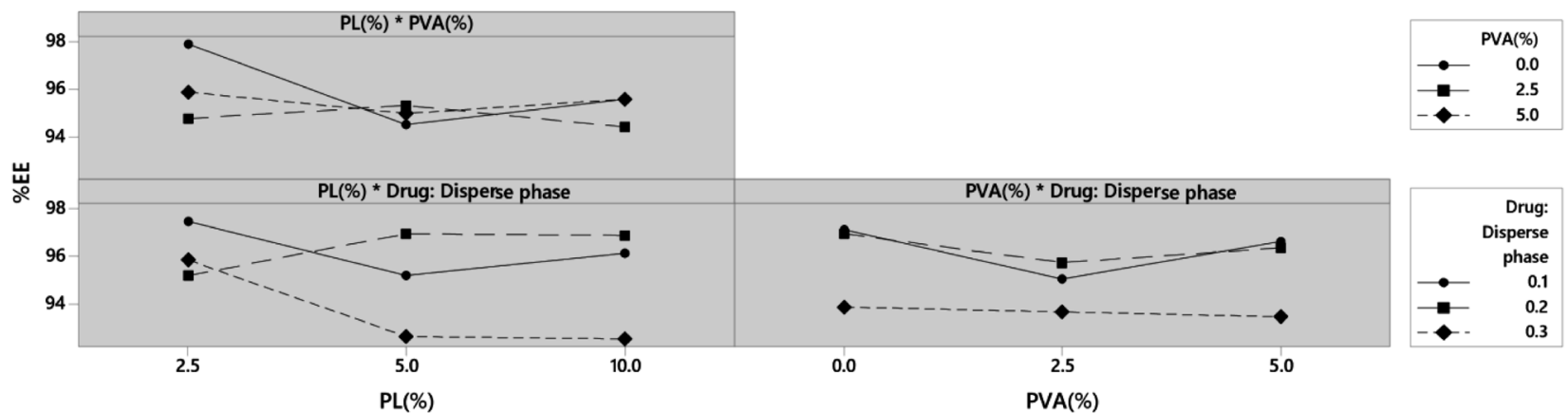

Fig. 3. Main Effect Plot, Interaction Plot and Pareto Plot of PL407 Concentration, PVA Concentration and Drug to Disperse Phase Ratio on \%EE for CB Loaded Cubosome Dispersions

nanochannels of the cubic nanoparticles were responsible and contributed to the slowing down of the release in the second phase. ${ }^{44,45)}$ This pattern can be highly advantageous for the treatment of patients with high risk of myocardial infarction and stroke as the initial rapid drug release phase is important to achieve high $\mathrm{CB}$ concentration in a very short time, while the slow steady state release of the remaining product would provide successful drug delivery along the time. Concurrently, the initial burst release of the drug will be well tolerated as higher doses (up to $600 \mathrm{mg}$ ) of $\mathrm{CB}$ were previously reported in literature and were well tolerated with no adverse effects. ${ }^{46)}$

Buffer transition release study was used to assess optimized cubosome dispersion CL1 compared to pure CB to mimic the gastrointestinal conditions and to assess the release of the drug along stomach and intestinal $\mathrm{pH}$ where it will be absorbed. According to previous studies which concluded maximum gastric emptying time of $2 \mathrm{~h}^{47}$ and maximum small intestine transit time $6 \mathrm{~h},{ }^{48}$ ) at which the formula is expected to fully release the drug. ${ }^{49)}$ Therefore, the release study was conducted for $2 \mathrm{~h}$ in gastric $\mathrm{pH}(1.2)$ and for $6 \mathrm{~h}$ in intestinal $\mathrm{pH}(6.8)$.

As evident in Fig. 5, CB \% released in both formulas has reached $100 \%$ in gastric $\mathrm{pH}$ as $\mathrm{CB}$ is freely soluble in acidic medium. This is expected as CB is a week base with $\left(\mathrm{p} K_{\mathrm{a}}=4.5\right)$, which causes its ionization in lower $\mathrm{pH}^{39)}$ however, it will subsequently hinder its absorption. ${ }^{6}$ Upon increasing $\mathrm{pH}$ to 6.8 to mimic the intestinal $\mathrm{pH}, \%$ released of $\mathrm{CB}$ significantly $(p<0.05)$ dropped to $66.82 \pm 4.12 \%$ after $6 \mathrm{~h}$ due to formation of unionized free base which have poor solubility. ${ }^{50)}$ On the contrary, optimized cubosome dispersion CL1 preserved \%released of $\mathrm{CB}$ at $95.66 \pm 1.87 \%$ ( $p>0.05)$ which can be attributed to the high affinity of $\mathrm{CB}$ to the lipid layer of cubosomes due to its hydrophobic nature ${ }^{39)}$ thereby retaining $\mathrm{CB}$ in solubilized state ${ }^{13,19)}$ and preventing its precipitation.
These findings are further endorsed by similarity factor higher lower than 50 ( $\mathrm{f} 2=14$ ) which confirm the success of cubosome dispersion to enhance the in vitro release of $\mathrm{CB}$ in simulated GIT conditions and prevent $\mathrm{CB}$ precipitation in the intestinal $\mathrm{pH}$.

By inspection of the main effects plot (Fig. 6A) of various variables on drug release $\left(\% \mathrm{Q}_{15}\right)$, it is observed that increasing PVA concentration insignificantly affected $\% \mathrm{Q}_{15}$. On the other hand increasing the ratio of drug to disperse phase significantly decreased $\% \mathrm{Q}_{15}$. This was expected if one considered the increase in particle size discussed earlier which resulted in a decrease in the surface area available for drug release. ${ }^{37)}$ Nevertheless, the concentration of PL407 had the most significant $(p<0.05)$ effect on the $\% \mathrm{Q}_{15}$ as shown in Pareto plot in Fig. 6B with $\% \mathrm{Q}_{15}$ decreases as PL407 increases. This can be ascribed to the coexistence of both Pn3m (CD) structure in which PL407 molecules are mostly absorbed on the surface with few PL407 forming the internal region of the cubosomal particles and Im3m (CP) structure in which the excess PL407 was incorporated with GMO in the inner core after the saturation of the cubosomes surface. As the PL407 increases, a phase transition takes place from $\mathrm{CD}$ to $\mathrm{CP}$ with restricted and lower drug release efficiency than $\mathrm{CD}^{38,51,52)}$ Furthermore, the two way ANOVA shows insignificant interactions $(p>0.05)$ between the tested factors as shown in Fig. 6C.

Based on the optimization of the factorial design and the desirability index (Table 3), formula CL1 showed the highest desirability index $(D=0.867)$ among the other formulas and the best attributes of high drug content and highest $\% \mathrm{Q}_{15}$ of $\mathrm{CB}$ release in intestinal $\mathrm{pH}$ (Fig. 4A). This can be ascribed to its low ratio of drug to disperse phase $(1: 10)$ and low concentration of PL407 (2.5\%) which proved through the analysis of the factorial design to be favourable formulation aspects for CB cubosomal dispersions. On the contrary, CL27 exhibited 

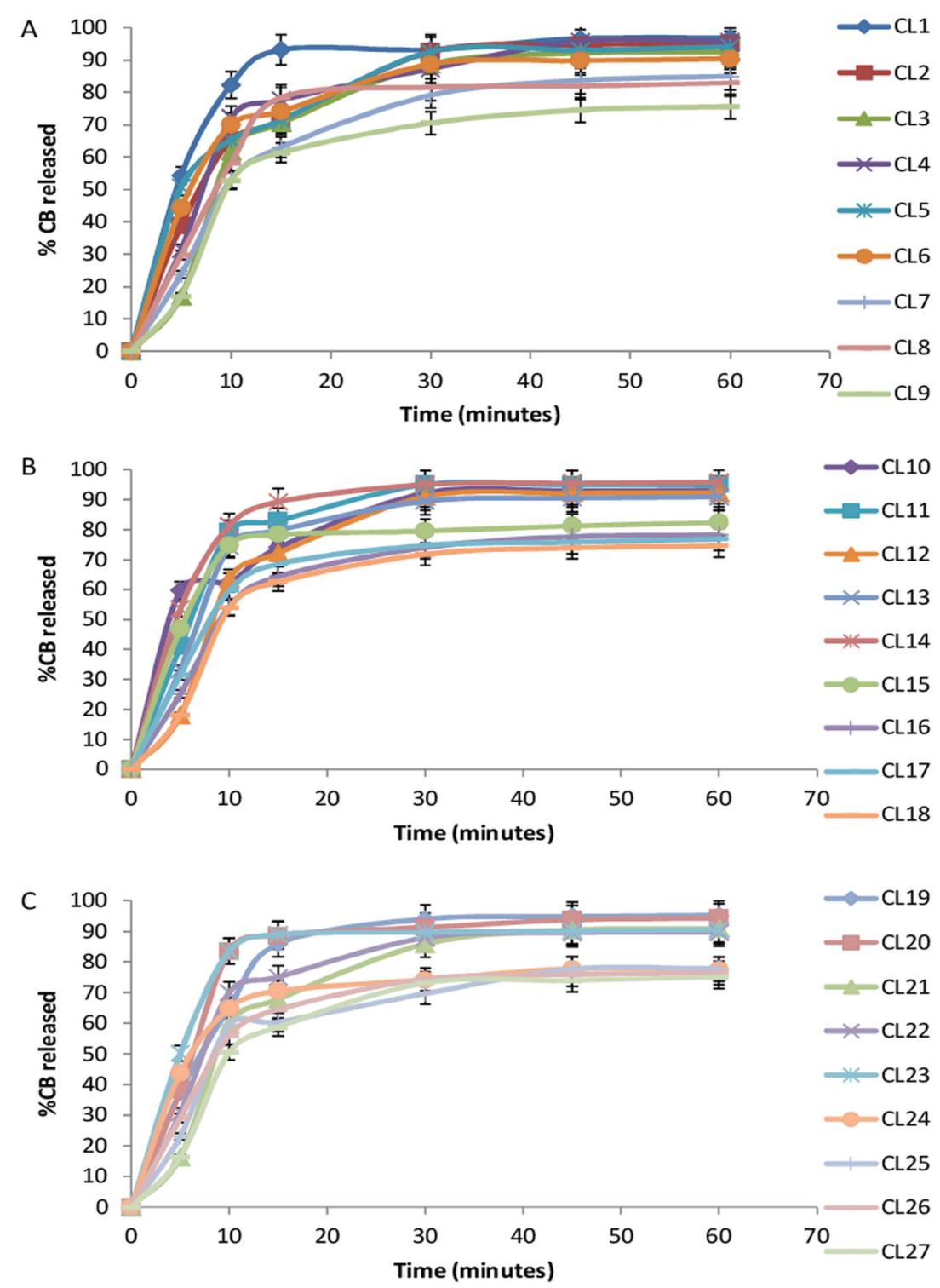

Fig. 4. Drug Release Profiles of CB Loaded Cubosome Dispersion in Phosphate Buffer pH 6.8 Containing $1 \%$ SLS at $37 \pm 0.5^{\circ} \mathrm{C}(n=6)$ for (A) CL1 to CL9, (B) CL10-CL18 and (C) CL19-CL27 and Free Drug

(Color figure can be accessed in the online version.)

the lowest desirability $(\mathrm{D}=0.121)$ and the lowest $\mathrm{CB}$ release $\left(\% \mathrm{Q}_{15}=58.71 \pm 7.23\right)$ as shown in Fig. $4 \mathrm{C}$. These results were consistent with CL27composition of high ratio of drug to disperse phase $(3: 10)$ and high PL407 concentration (10\%) as well which proved to be unfavourable for CB loaded cubosomes formulation according to the statistical design. Thus, CL1 was further progressed to in vivo clinical assessment of its antihaemostatic effect through bleeding time measurement.

In Vivo Bleeding Time $\mathrm{CB}$, being a BCS class II drug, its poor solubility in the intestine is a limiting step in its absorption in vivo. Thus, increasing its release in intestinal $\mathrm{pH}$ in vitro will probably lead to enhanced drug levels in blood stream. $^{27,53)}$ However, enhanced $\mathrm{CB}$ intestinal release is not adequate to prove the potential success of the prepared cubosomal dispersion in vivo. Thus assessing the effect on blood clotting and haemostasis of the prepared formula in vivo is of equal if not much importance for consideration when assessing cubosomal dispersions. Bleeding time has been a mainstay

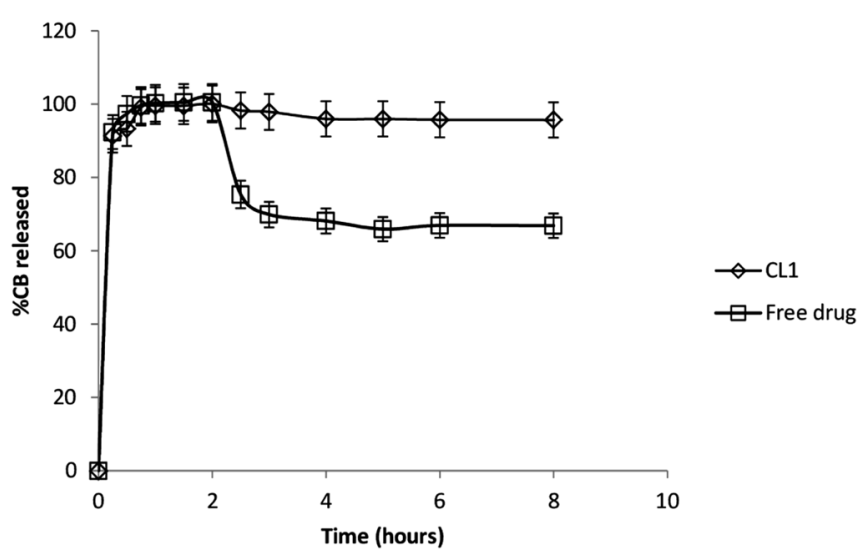

Fig. 5. Drug Release Profiles of CB Optimized Cubosome Dispersion CL1 and Free CB Powder in Buffer Transition System (pH 1.2 for $2 \mathrm{~h}$ Then $\mathrm{pH} 6.8$ Containing $1 \%$ SLS for $6 \mathrm{~h})$ at $37 \pm 0.5^{\circ} \mathrm{C}(n=6)$ 
A

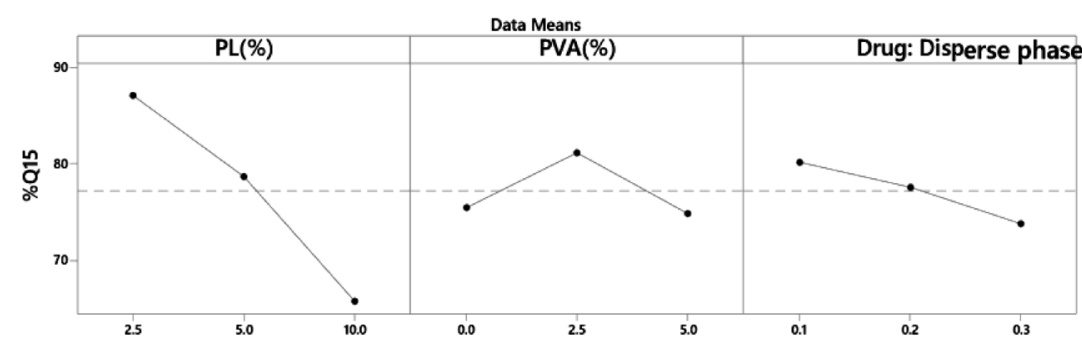

B

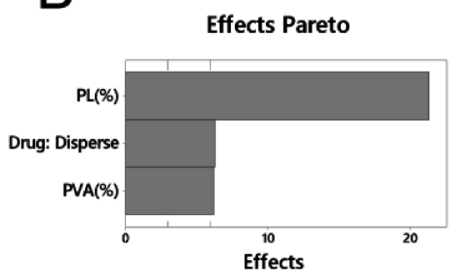

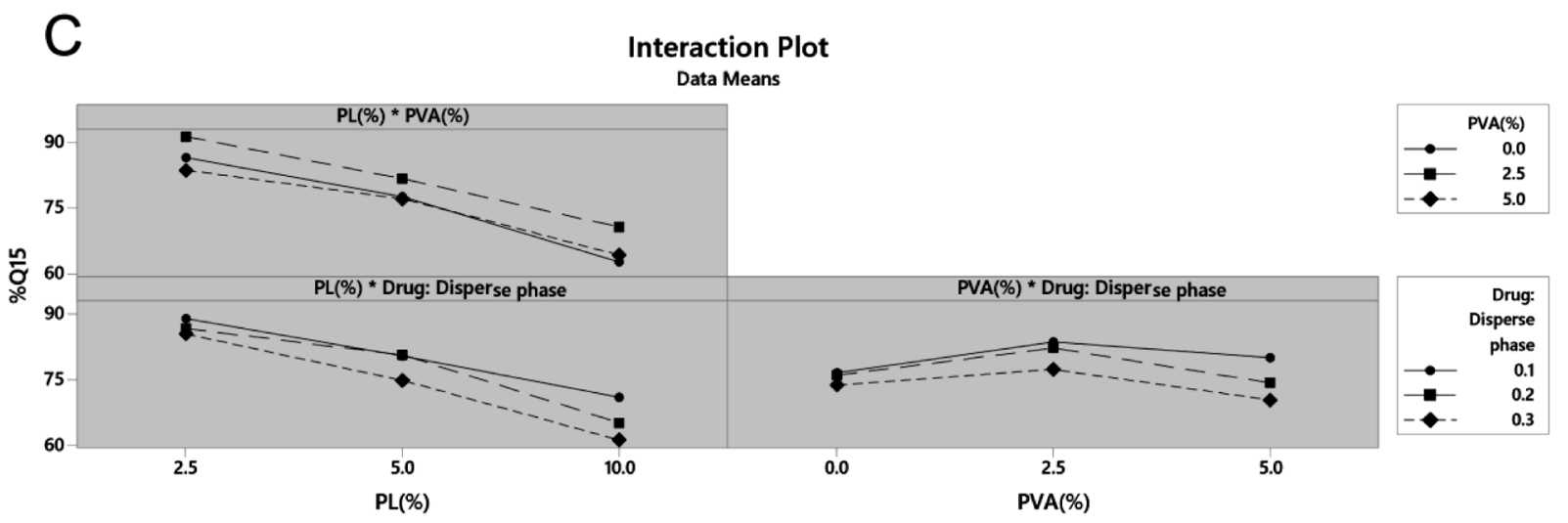

Fig. 6. Main Effect Plot, Interaction Plot and Pareto Plot of PL407 Concentration, PVA Concentration and Drug to Disperse Phase Ratio on $\% Q_{15}$ for CB Loaded Cubosome Dispersions

clinical assessment approach for measuring the haemostasis and blood clotting behaviour which was endorsed as a direct reflection technique to the in vivo effectiveness and the adequacy of the measurement to the antithrombotic activity. ${ }^{32)}$

It is evident from Fig. 7 that cubosome dispersion CL1 had significantly $(p<0.05)$ increased BT $(628.47 \pm 6.12 \mathrm{~s})$ compared to Plavix ${ }^{\circledR}(412.43 \pm 7.97 \mathrm{~s})$ thereby confirming the increased antihaemostatic action of cubosome nanoparticles compared to commercial market. Both CL1 and Plavix ${ }^{\circledR}$ treatments were significantly $(p<0.05)$ different from the control group (202.12 $\pm 4.21 \mathrm{~s})$ thus ensuring that the recorded differences

CL1 significantly increased BT compared to Plavix ${ }^{\circledR}$ can be directly attributed to the increased CB levels in blood stream, which in turn can be directly correlated to the enhanced $\mathrm{CB}$ release and its rapid release and absorption in the intestine mediated by the developed cubosome nanoparticles. ${ }^{13,32,54)}$ This can be ascribed to the digestion of GMO lipid by the pancreatic lipases to form mixed micelles, cubic, hexagonal and vesicular phases. ${ }^{13,20)}$ At this environment, the drug released into the gastrointestinal fluid will be solubilized in the micelles or mixed micellar structures thereby preventing precipitation in the intestinal $\mathrm{pH}$ and will be ready for immediate absorption. ${ }^{15)}$

Based on the evidence in this study, cubosome nanoparticles offer a promising technique for increasing $\mathrm{CB}$ solubility in intestinal $\mathrm{pH}$, the main site of its absorption with excellent entrapment efficiency and adequate particle size. The current work demonstrated that the enhanced release of $\mathrm{CB}$ cubosomal dispersion has indeed associated with an in vivo enhanced blood flow compared to marketed product Plavix ${ }^{\circledR}$ in rabbits, thereby endorsing the merits of cubosomes as a

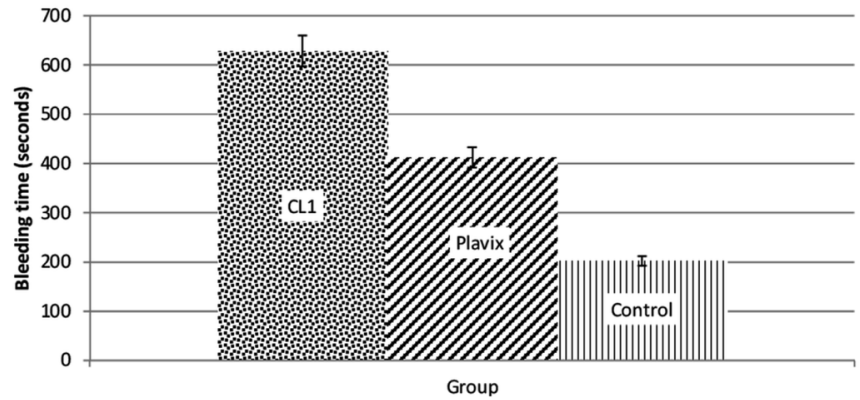

Fig. 7. Histogram Comparing BT of Optimized CB Loaded Cubosomal Dispersion CL1, Plavix ${ }^{\circledR}$ and Control in Rabbits $(n=6)$

promising drug delivery system for $\mathrm{CB}$. The factorial design applied in this study demonstrated that the ratio of the drug to the disperse is the most significant and contributing factor affecting the attributes of the prepared cubosome nanoparticles followed by PL407 concentration making these factors a critical decision in the formulations of these systems. Unlike PVA concentration which proved to be insignificant during cubosome nanoparticles formulations. These findings pave the road for cubosome nanoparticles to offer a new platform for enhancing the release properties of BCS class II drugs and improving their in vivo performance. Further research in this area is recommended on different drugs to explore the cubosomes potential to enhance oral bioavailability of BCS class II drugs in vitro and in vivo.

Conflict of Interest The authors declare no conflict of interest. 


\section{References}

1) Kim Y. I., Kim K. S., Suh K. H., Shanmugam S., Woo J. S., Yong C. S., Choi H. G., Int. J. Pharm., 415, 129-139 (2011).

2) Sangkuhl K., Klein T. E., Altman R. B., Pharmacogenet. Genomics, 20, 463-465 (2010).

3) Tan C., Degim I. T., Pharm. Dev. Technol., 17, 242-250 (2012).

4) Savi P., Herbert J., Pflieger A., Dol F., Delebassee D., Combalbert J., Defreyn G., Maffrand J., Biochem. Pharmacol., 44, 527-532 (1992).

5) Farid N. A., Kurihara A., Wrighton S. A., J. Clin. Pharmacol., 50, 126-142 (2010).

6) Bali D. E., Osman M. A., El Maghraby G. M., Eur. J. Drug Metab. Pharmacokinet., 41, 807-818 (2016).

7) Lassoued M. A., Sfar S., Bouraoui A., Khemiss F., J. Pharm. Pharmacol., 64, 541-552 (2012).

8) Karaźniewicz-Łada M., Danielak D., Burchardt P., Kruszyna Ł., Komosa A., Lesiak M., Glówka F., Clin. Pharmacokinet., 53, 155164 (2014).

9) Wgih M. P., Patel J. S., Int. J. Pharm. Pharm. Sci., 2, 12-19 (2010).

10) Pouton C. W., Porter C., Adv. Drug Deliv. Rev., 60, 625-637 (2008).

11) Hartnett T. E., O’Connor A. J., Ladewig K., Expert Opin. Drug Deliv., 9, 1-15 (2015).

12) Boyd B., Int. J. Pharm., 260, 239-247 (2003).

13) Lai J., Chen J., Lu Y., Sun J., Hu F., Yin Z., Wu W., AAPS PharmSciTech, 10, 960-966 (2009).

14) Tayel S. A., El-Nabarawi M. A., Tadros M. I., Abd-Elsalam W. H., Drug Deliv., 23, 3266-3278 (2016).

15) Yang Z., Chen M., Yang M., Chen J., Fang W., Xu P., Int. J. Nanomedicine, 9, 327-336 (2014).

16) Ganem-Quintanar A., Quintanar-Guerrero D., Buri P., Drug Dev. Ind. Pharm., 26, 809-820 (2000).

17) Kwon T. K., Hong S. K., Kim J.-C., J. Ind. Eng. Chem., 18, 563-567 (2012).

18) Yang Z., Tan Y., Chen M., Dian L., Shan Z., Peng X., Wu C., AAPS PharmSciTech, 13, 1483-1491 (2012).

19) Ali M. A., Kataoka N., Ranneh A. H., Iwao Y., Noguchi S., Oka T., Itai S., Chem. Pharm. Bull., 65, 42-48 (2017).

20) Nasr M., Dawoud M., J. Drug Deliv. Sci. Technol., 35, 106-113 (2016).

21) "The United States pharmacopeia 39/National Formulary 34," The United States Pharmacopeial Convention Inc., Rockville, MD, 2016.

22) Morsi N. M., Abdelbary G. A., Ahmed M. A., Eur. J. Pharm. Biopharm., 86, 178-189 (2014).

23) Abdelwahed N. A. M., Ahmed E. F., El-Gammal E. W., Hawas U. W., 3 Biotech., 4, 533-544 (2014).

24) Elkomy M. H., El Menshawe S. F., Eid H. M., Ali A. M., Drug Dev. Ind. Pharm., 43, 531-544 (2017).

25) Derringer G., Suich R., J. Qual. Technol., 12, 214-219 (1980).

26) Thapa R. K., Baskaran R., Madheswaran T., Kim J. O., Yong C. S., Yoo B. K., J. Drug Deliv. Sci. Technol., 22, 479-484 (2012).

27) Jassim Z. E., Hussein A. A., Int. J. Pharm. Pharm. Sci., 6, 838-851 (2014).

28) Lee Y. S., Song J. G., Lee S. H., Han H. K., Drug Deliv., 24, 17311739 (2017).
29) Feng D., Peng T., Huang Z., Singh V., Shi Y., Wen T., Lu M., Quan G., Pan X., Wu C., Pharmaceutics, 10, E53 (2018).

30) Javadzadeh Y., Shariati H., Movahhed-Danesh E., Nokhodchi A., Drug Dev. Ind. Pharm., 35, 243-251 (2009).

31) Moore J. W., Flanner H. H., Pharm. Technol., 20, 64-74 (1996).

32) Wong P. C., Crain E. J., Watson C. A., Jiang X., Hua J., Bostwick J. S., Martin L., J. Cardiovasc. Pharmacol., 49, 316-324 (2007).

33) Molero L., Lopez-Farre A., Mateos-Caceres P. J., Fernandez-Sanchez R., Luisa Maestro M., Silva J., Rodriguez E., Macaya C., Br. J. Pharmacol., 146, 419-424 (2005).

34) Shah J. C., Sadhale Y., Chilukuri D. M., Adv. Drug Deliv. Rev., 47, 229-250 (2001).

35) Esposito E., Cortesi R., Drechsler M., Paccamiccio L., Mariani P., Contado C., Stellin E., Menegatti E., Bonina F., Puglia C., Pharm. Res., 22, 2163-2173 (2005).

36) Barnard A. S., Zapol P., J. Chem. Phys., 121, 4276-4283 (2004).

37) Dora C. P., Singh S. K., Sanjeev Kumar A., Datusalia K., Deep A., Acta Pol. Pharm., 67, 283-290 (2010).

38) Esposito E., Eblovi N., Rasi S., Drechsler M., Gregorio G. M. D., Menegatti E., Cortesi R., AAPS PharmSciTech, 5, 1-15 (2003).

39) Remko M., Remkova A., Broer R., Int. J. Mol. Sci., 17, 388 (2016)

40) Sharma N., Madan P., Lin S., Asian J. Pharm. Sci., 11, 404-416 (2016).

41) Nasr M., Ghorab M. K., Abdelazem A., Acta Pharm Sin. B, 5, 79-88 (2015).

42) Swain S., Patra C. N., Rao M. E. B., "Pharmaceutical Drug Delivery Systems and Vehicles," Woodhead Publishing Ltd., India, 2016.

43) Magenheim B., Levy M. Y., Benita S., Int. J. Pharm., 94, 115-123 (1993).

44) Clogston J., Caffrey M., J. Control. Release, 107, 97-111 (2005).

45) Boyd B. J., Whittaker D. V., Khoo S. M., Davey G., Int. J. Pharm. 309, 218-226 (2006).

46) Herbert J. M., Frehel D., Vallee E., Kieffer G., Gouy D., Berger Y., Necciari J., Defreyn G., Maffrand J. P., Cardiovasc. Drug Rev., 11, 180-198 (1993).

47) Vasavid P., Chaiwatanarat T., Pusuwan P., Sritara C., Roysri K., Namwongprom S., Kuanrakcharoen P., Premprabha T., Chunlertrith K., Thongsawat S., Sirinthornpunya S., Ovartlarnporn B., Kachintorn U., Leelakusolvong S., Kositchaiwat C., Chakkaphak S., Gonlachanvit S., Neurogastroenterol. Motil., 20, 371-378 (2014).

48) Davis S. S., Hardy J. G., Fara J. W., Gut, 27, 886-892 (1986).

49) Rawat M., Saraf S., Saraf S., AAPS PharmSciTech, 8, 289-297 (2007).

50) Zupancic V., Smrkolj M., Benkic P., Simonic I., Plevnik M., Ritlop G., Kristl A., Vrecer F., Acta Chim. Slov., 57, 376-385 (2010).

51) Nakano M., Sugita A., Matsuoka H., Handa T., Langmuir, 17, 3917-3922 (2001)

52) Zhao Y., Zhang J., Zheng L. Q., Li D. H., J. Disper. Sci. Technol., 25, 795-799 (2004)

53) Papich M. G., Martinez M. N., AAPS J., 17, 948-964 (2015).

54) Hoffmann P., Bernat A., Savi P., Herbert J. M., J. Pharmacol. Exp. Ther., 286, 670-675 (1998). 